\title{
THE REVIEW ON AUTOMATIC LICENSE PLATE RECOGNITION
}

\section{(ALPR)}

\author{
Rinku Solanki1 ${ }^{1}$, Rajesh Kumar Rai $^{2}$,Teena Raikwar ${ }^{3}$ \\ ${ }^{1}$ M.tech, student, digital communication, NRI-IST, Bhopal, Madhya Pradesh, India \\ ${ }^{2}$ Professor, Head of the department of E. C, NIRT, Bhopal, Madhya Pradesh, India, \\ ${ }^{3}$ Assistant professor, electronics \& communication dept., (NRI-IST, Bhopal), Madhya Pradesh, India \\ rinkusolanki86@gmail.com,raj.rai1008@gmail.com,t_raikwar.2006@yahoo.co.in
}

\begin{abstract}
Abstract explanation should be Times New Roman, Font Size 10, Single line spacing, Italic, Text alignment should be justify, should contain at least 250 words. Abstract explanation should be Times New Roman, Font Size 10, Single line spacing, Italic, Text alignment should be justify, should contain at least 250 words. Abstract explanation should be Times New Roman, Font Size 10, Single line spacing, Italic, Text alignment should be justify, should contain at least 250 words. Abstract explanation should be Times New Roman, Font Size 10, Single line spacing, Italic, Text alignment should be justify, should contain at least 250 words. Abstract explanation should be Times New Roman, Font Size 10, Single line spacing, Italic, Text alignment should be justify, should contain at least 250 words. Abstract explanation should be Times New Roman, Font Size 10, Single line spacing, Italic, Text alignment should be justify, should contain at least 250 words.
\end{abstract}

Keywords: Key word1, Key word2, Key word3, and Key word4 etc... - $* * *$

\section{INTRODUCTION}

The purpose of this paper is to provide researchers a systematic survey of existing ALPR research by categorizing existing methods according to the features they used, by analysing the pros/cons of these features, and by comparing them in terms of recognition performance and processing speed, and to open some issues for the future research.

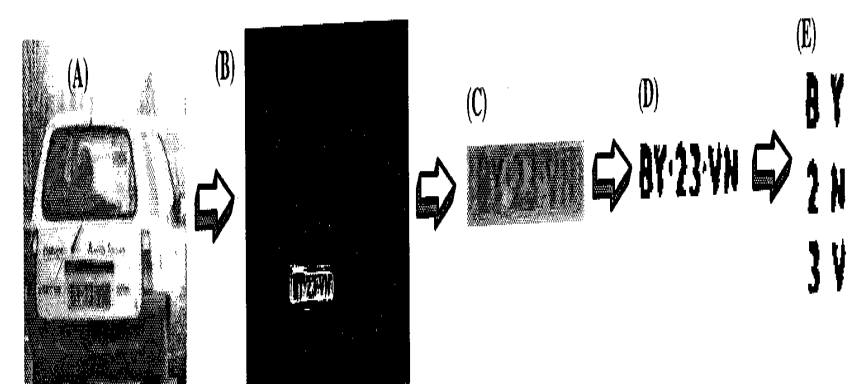

Figure1: An Example of Extraction of Number Plate

Basic block diagram of the ALPR system is shown in fig 2.for above steps different techniques used by different author which are studied in literature review. An example of the number plate extraction is given by figure (1).by this figure block diagram is easily understand, in this figure all steps of block diagram is shown by indicating number A,B,C,D,E.
Automatic license plate recognition (ALPR) applies image processing and character recognition technology to identify vehicles by automatically reading their number plates. And this system mainly divides in three steps:

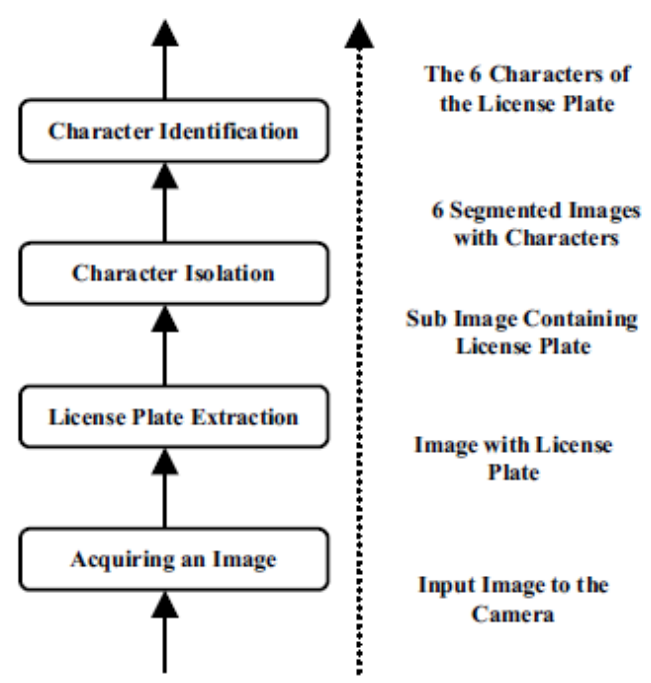

Figure2: Basic Block diagram Of Alpr System

Step(1)Acquiring an Image, in image acquisition explained that from where images are acquire Image can be input to the system by different methods by analog camera, or by digital 
cameras, but nowadays digital technology has their advantages so better input method is by digital cameras or by direct digital photos.

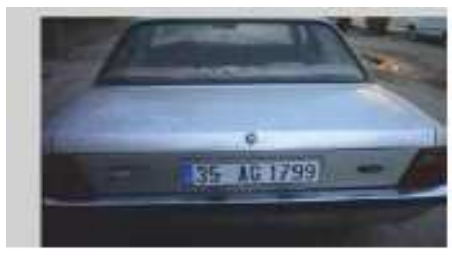

Figure3: captured image

Step (2) License Plate Extraction by whole capturing image we having license plate covered by background of vehicle body, so by this step only plate are is extracted from whole body. Our task now is to identify the region containing the license plate. In this experiment, two features are defined and extracted in order to decide if a candidate region contains a license plate or not, these features are

\section{Aspect ratio:}

The aspect ratio is defined as the ratio of the width to the height of the region.

$$
\text { Aspect Ratio }=\text { width } / \text { height }
$$

Since the minimum enclosing rectangle (MER) of the object region can be computed via rotating the region in previous section, the dimension of the objects MER can be taken as the width and the height of the region

\section{Edge Density}

Applying the above feature to filter the segmented regions, a lot of no license plate regions can be removed. However, there are still many candidate regions left which take similar rectangularity and aspect ratio features as the license plate regions do, such as often the head lights. Considering that the license plate regions generally take higher local variance in its pixelse values due to the presence of characters, an important feature to describe license plate region is local variance, which is quantized using the edge density. So extracted plate region example shown in figure 4.

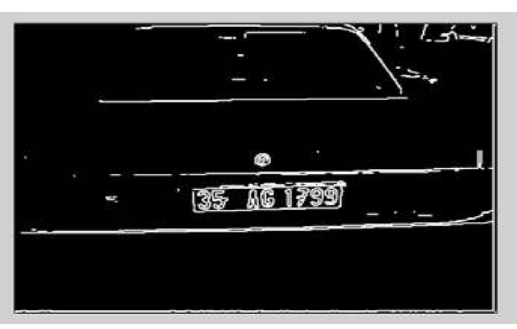

Figure4: license plate extraction
Step (3) Character Isolation by this step characters on license plate are segmented and identify. This step is the most important step in license plate recognition because all further steps rely on it. This is the second major part of the License Plate detection algorithm. There are many factors that cause the character segmentation task difficult, such as image noise, plate frame, rivet, space mark, plate rotation and illumination variance. We here propose the algorithm that is quite robust and gives significantly good results on images having the above mentioned problems. for the segmentation preprocessing is required by conversion to gray scale and binarization. Different algorithms are used for segmentation which are explained further later in literature review. Segmented license plate example is given in figure 5

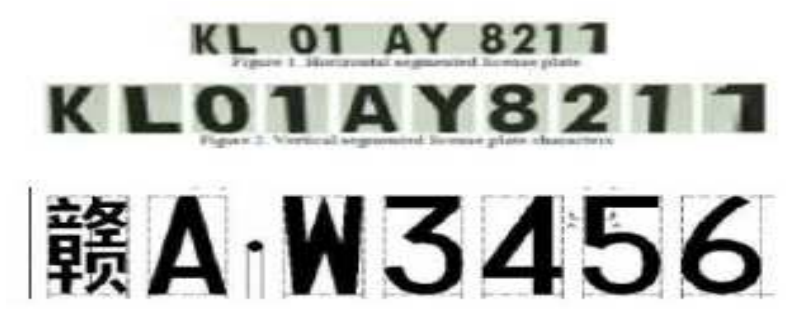

Figure5: character isolation

Step (4) Character Identification by this step final result is founded. Consider figure6 as an final extracted license plate.

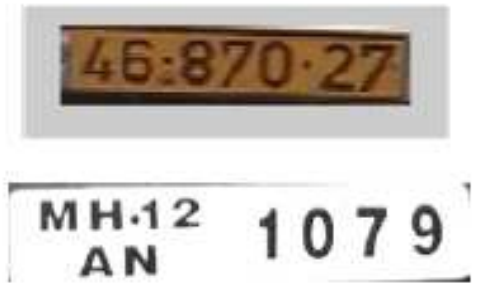

Figure6: character identification

The variations of the plate types or environments cause challenges in the detection and recognition of license plates. They are summarized as follows:

1) Location: Plates exist in different locations of an image.

2) Quantity: An image may contain no or many plates.

3) Size: Plates may have different sizes due to the camera distance and the zoom factor.

4) Colour: Plates may have various characters and background colours due to different plate types or capturing devices.

5) Font: Plates of different nations may be written in different fonts and language.

6) Occlusion: Plates may be obscured by dirt.

7) Inclination: Plates may be tilted.

8) Other: In addition to characters, a plate may contain frames and screws. 


\subsection{Environment variations:}

1) Illumination: Input images may have different types of illumination, mainly due to environmental lighting and vehicle headlights.

2) Background: The image background may contain patterns similar to plates, such as numbers stamped on a vehicle, bumper with vertical patterns, and textured floors.
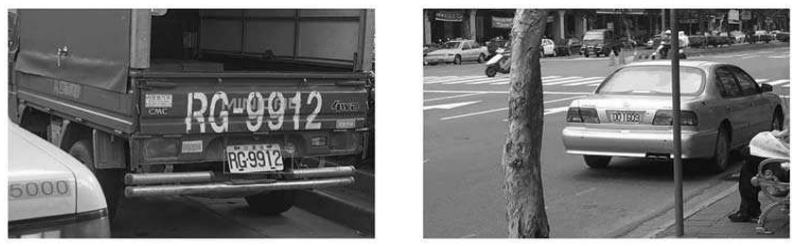

(a)
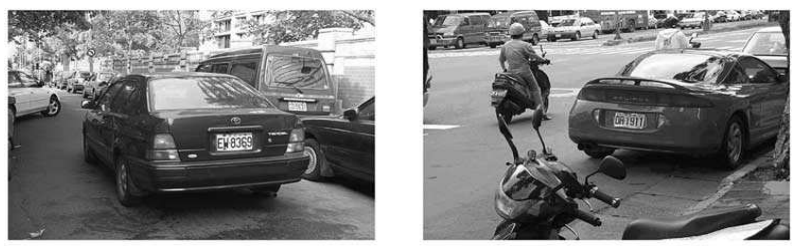

(b)
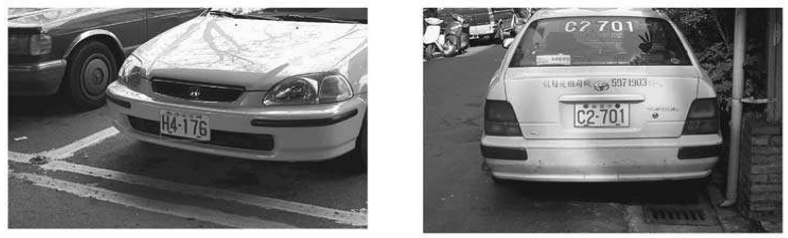

(c)
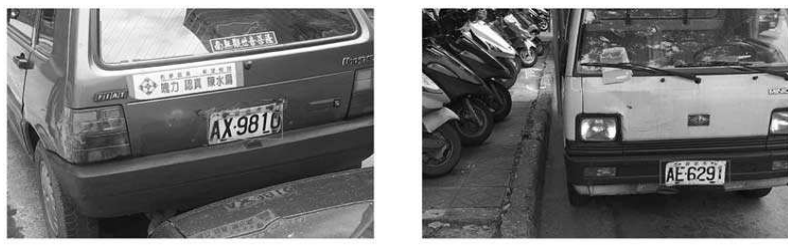

(d)

Figure7: Example images in the different condition :( a) Complex scenes. (b) Various environments. (c) Different illuminations. (d). Damaged license plates.

\section{LITERATURE REVIEW}

In the literature, many license plate detection algorithms have been proposed. Although license plate detection has been studied for many years, it is still a challenging task to detect license plates from different angles, partial occlusion, or multiple instances. License plate detection investigates an input image to identify some local patches containing license plates. Since a plate can exist anywhere in an image with various sizes, it is infeasible to check every pixel to locate it. Generally, it is preferable to extract some features from images and focus only on those pixels characterized by the license plate. Based on the involved features, traditional license plate detection methods can be classified into three categories: colour-based, edge-based, and texture-based. In what follows, we will review the related work in each category Colour-based approaches are based on the observation that some countries have specific colours in their license plates. It is intuitive to extract license plates by locating their colours in the images the collocation of license plate colour and character colour is used to generate an edge image. Then, it checks neighbours of pixels with a value within the license plate colour range to find candidate license plate regions Edge-based approaches are the most popular, with reliable performance in license plate detection. Generally, as a prior, license plate is characterized by a rectangular shape with a specific aspect ratio, and can be extracted by checking all possible rectangles in the image kinds of traditional locating methods, some other approaches based on local features have been proposed recently. A brief description of some of previous works is demonstrated in section of literature introduction.

In [1], license plate recognition methods are:(1)Image Acquisition: By digital camera (2) License Plate Extraction: *vertical edge detection by sobel algorithm *filtering by seed filling algorithm *vertical edge matching (3) Segmentation: (4) Character Recognition: * Normalization * Template matching using hamming distance approach. and by this paper referenced getting the result like: License Plate Extraction: 587/610, 96.22\% License Plate Segmentation: 574/610, 94.04\% License Plate Recognition :581/610, 95.24\%,and over all system efficiency: $95 \%$.this approach having some problem in extracting the plate, diplomatic cars and military vehicles, are not addressed since they are rarely seen. Detection only for white, black, red, and green colour plate or numbers.

By [2], (1) Extraction of plate region: edge detection algorithms and smearing algorithms (2) segmentation of Characters: smearing algorithms, filtering and some morphological algorithms (3)recognition of plate characters : template matching. Final output it is proved to be \%97.6 for the extraction of plate region, $\% 96$ for the segmentation of the characters and \%98.8 for the recognition unit accurate, giving the overall system performance $\% 92.57$ recognition rate .it having some limitation like it recognition of car license plate only, and This system is designed for the identification of Turkish license plates.

In[3], recognition steps are as follow:(1)Image Enhancement: by histogram equalization method (2)Structuring Elements : by thickening, (3) Hat transformations: which is use for contrast, enhancement(top has \& bottom has)setting (4) Morphological Operations like dilation and erosion (5) Plate region confirmation (6) Character Segmentation and Recognition by neuron implementation model by this reference 250 colour images were used for testing the technique, These results report a high accuracy rate of above $95 \%$.Although the technique is quite efficient enough to work very well in the real time 
environment but currently the technique proposed lays more emphasis on the accuracy of the overall system, while the some more work is to be done to make the technique more efficient.

In[4],recognition by(1) Target recognition: by using featuresalience theory, features of license plates( include shape, symmetry, height-to-width ratio, colour, texture, and spatial frequency, Character features include lines, blobs, aspect ratio of characters, distribution of intervals between characters ,and alignment of characters) (2license plate locating by Hough transform (HT). (3) Recognizing license characters by different steps like binarization, noise removal, and orientation adjustment, Optical Character Recognition. in this paper, the success rate for the identification with the set of 1144 license plates is $95.7 \%$. Combining this rate with the location success (97.3\%), the overall rate of success for our LPR algorithm is 93.1\%.As pointed out in the preceding sections, although this system is intended for the recognition of Chinese license plates only.

By[5], mainly focused on Edge Detection(Sobel Edge Detection)technique and then filtering of noise by Median Filter, Smoothing, Connector, Masking, and then Colour Conversation is done. we can see that the detection is not that clear and proper, which we find, is due to improper light segment or varying illumination effects. And all over system result is not mention in this paper.

In the reference [6], The proposed algorithm consists of three major parts: Extraction of plate region, segmentation of characters and recognition of plate characters. For (1) extracting the Plate region, edge detection algorithm and vertical projection method are used.(2) in segmentation part filtering, thinning and vertical and horizontal projection are used. And finally, (3)chain code concept with different parameter is used for recognition of the characters. The performance of the proposed algorithm has been tested on real images. Total Vehicles Images 150(tested under sunny, cloudy, daytime, nigh time, rainy days...etc atmosphere), Extracted license plates147 Unsuccessful Extraction 3 .and final system Efficiency: $98 \%$. The proposed method is mainly designed for real-time Malaysian license plate, and can be readily extended to cope with license plates of other countries, especially those using Latin characters.

By[7],involve three approaches: (1)in plate localization Noise alleviation, Changing colour space, Intensity dynamic range modification, Edge detection ,Separating objects from background, Finding connected component ,Candidate selection, all above process are used (2) in segmentation part multistage model are used.(Improvement, Rotation, Binarization, Segmentation, Preparation, (3)for the recognition artificial 1 1Feed forward neural network is used. The method achieved accuracy over $91 \%$ for localizing plates. The recognition system implemented by neural networks after segmentation of characters in image plate identify alphabets and numbers separately and achieve an accuracy over $97 \%$ and 94\% respectively for each. Advantage of this approach is The image database includes images of various vehicles with different background and slop under varying illumination condition and the disadvantage is detection only for English and Parisian number plate.

In[8] For the Number plate recognition first image conversion in binary and apply to neural network, and apply $\mathrm{mpl}$ algorithm, then detection individual symbol, by matrix mapping, and Training by this approach obtained $96.53 \%$ average recognition rate using double hidden layer and 94\% using single hidden layer. The captured image 2-3 meters taken away from the cameras

By[9] (1)Pre-processing of Image by histogram equalization(2)Extraction of plate region by edge detection algorithm( canny operator) and Plate Area Detection by various morphological operations (3)Segmentation of characters by *connected component *bounding box method, * Median filter, all above methods. and observed final result as Extraction :71/78 which gives $91.02 \%$ efficiency ,Segmentation 69/78 which gives $88.46 \%$ efficiency. overall accuracy of our system is $89.74 \%$.proposed method is sensitive to the angle of view, physical appearance and environment conditions.

By[10] Given All 3 process by 2D Haar after the discrete wavelet Transform technique : (1)locate and extract the license-plate (2)train of the license-plate (3)real time scan recognize of the license-plate by this paper result shown are as given, Vehicle recognition number:100,Recognition number of successful:93 ,Recognition number failed :7, Recognition rates (\%) :93.0\%.advantage of this approach is Haar Discrete Wavelet Transform are that it each time transform only needs $1 / 4$ of the original image. Hence, this method can fast execution speed. and the Disadvantage is that in this paper only specified cameras used like Using the CASIO EXILIM, 10.1 MEGA PIXELS DIGITAL CAMERA EX-S10, adjusting the resolution $480 \times 640$ for photography vehicle license plates,

In[11] detection steps are :(1)Image acquisition by capturing an image of a vehicle from video (2)License plate detection extraction, by Spectral Analysis Approach and Connected Component Analysis (3)extract the region of license plate process use spectral analysis (4)Character segmentation use Connected component analysis approach and SVM feature extraction techniques. the advantage of this approach is success full recognition of a moving vehicle.

$\mathrm{By}$ [12] (1)PVW approach is used in this orientation, ratio of scale to character height, and relative position in the character region are done by clustering (2)visual word matching by comparing the extracted SIFT features and histogram approach 
is used (3) for license plate location A bounding box will be estimated to encompass license plate by determining the upper, lower, left, and right bounding lines sequentially. This technique achieves a 93.2\% "true" detection rate. This approach can also be extended to the detection of logos and trademarks .The weakness of this approach is that it may fail when the license plate resolution is too low, or when the distortion from the observation angle is too severe.

Approach explained in[13] that, (1) detect a license plate region by vertical or a horizontal edge based method (2) preprocessing: is also needed in this approach .so first converted in to binary image then eliminate noise using morphological operation (3) character segmentation by thresholding method (4) feature extraction and character recognition by Euler number formation.Advantage of this approach that skew ness is not present in the detected vehicle number plate compare to other methods and Disadvantage is it limits the efficiency of the total system.

By[14] (1)plate extraction by Mathematical Morphology approach and then ,Dilation and Erosion is apply to image,(2)segmentation by Structuring Elements approach, Meredian Filter technique and Edge Detection Methods are use,(3)character extraction by Pre-processing, Text/non-text classification approach .result derived by this paper is as given, Real Time Data:100,Images correctly Detected:93, Results Accuracy $93 \%$.and it says that Very much damaged plate cannot be recognize.

\begin{tabular}{|l|l|} 
'| YEARMMONTH & $\%$ EFFICENCY \\
\hline 2003 & 95 \\
\hline $2005 \mathrm{NOV}$ & 92.57 \\
\hline $2008 \mathrm{JNN}$ & 95 \\
$2009 \mathrm{SEPT}$ & 93.1 \\
2010 & 98 \\
\hline $2011 \mathrm{JULY}$ & 94 \\
\hline $2011 \mathrm{JLY}$ & 95 \\
\hline $2011 \mathrm{DEC}$ & 89.74 \\
\hline $2012 \mathrm{APRL}$ & 93 \\
\hline $2012 \mathrm{EPT}$ & 93.2 \\
\hline 20120CT & 93 \\
\hline
\end{tabular}

Figure8: table of \%efficiency/month or year

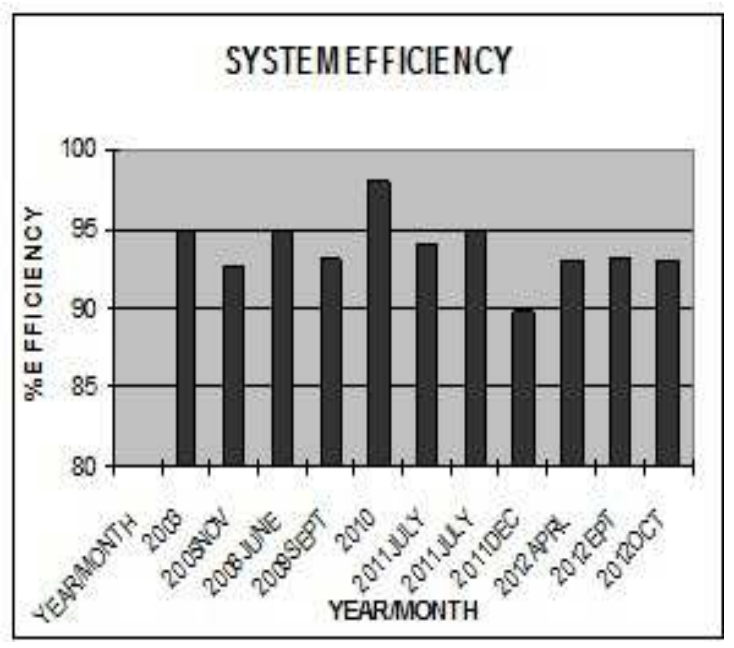

Figure9: Overall system \% efficiency/year

As per literature study all over system efficiency per month/year as given by chart figure9 which is vary according to methods for different steps for reconditioning of license plate, by the given plot we can observe percentage efficiency of system as per given data. As per given data in studied references making of table which shows \%efficiency per month/year, so consider the table for plot.

\section{CONCLUSIONS}

In general, an ALPR system consists of four processing stages. In the image acquisition stage, some points have to be considered when choosing the ALPR system camera, such as the camera resolution and the shutter speed. In the license plate extraction stage, the license plate is extracted based on some features such as the colour, the boundary, or the existence of the characters. In the license plate segmentation stage, the characters are extracted by projecting their colour information, by labelling them, or by matching their positions with template. Finally, the characters are recognized in the character recognition stage by template matching, or by classifiers such as neural networks and fuzzy classifiers. Automatic license plate recognition is quite challenging due to the different license plate formats and the varying environmental conditions. There are numerous ALPR techniques have been proposed in recent years.

\section{ACKNOWLEDGEMENTS}

I would like to thank professor rajesh kumar rai who is lecturer in our college they give me proper and perfect guidance in this literature paper .another person who give me a direction is $\mathrm{Mr}$. Rajesh nema sir, He is H.O.D. of our college. by the strict way they take work regularly from me and that's why I lots of thankful to them. i can say that because of strict nature of nema sir I can complete my work properly. 


\section{REFERENCES:}

[1] Muhammad Sarfraz, Mohammed Jameel Ahmed, and Syed A. Ghazi "Saudi arebian licence plate recognition system", International Conference on Geometric Modeling and Graphics (GMAG'03), 2003.

[2] Serkan Ozbay, and Ergun Ercelebi," Automatic Vehicle Identification by Plate Recognition", Processing of world academy of science engineering and technology vol9, ISSN 1307-6884, november 2005.

[3] Humayun Karim Sulehria, Ye Zhang, Danish Irfan, Atif Karim Sulehria," Vehicle Number Plate Recognition Using Mathematical Morphology and Neural Networks", WSEAS TRANSACTIONS on COMPUTERS, Volume 7, ISSN: 11092750, Issue 6, June 2008.

[4] Zhen-Xue Chen, Cheng-Yun Liu, Fa-Liang Chang, and Guo-You Wang," Automatic License-Plate Location and Recognition Based on Feature Salience", IEEE Transaction on vehicle technology, VOL. 58, NO. 7, september 2009.

[5] Dr. P.K.Suri, Dr. Ekta Walia, Er. Amit Verma," Vehicle Number Plate Detection using Sobel Edge Detection Technique", International Journal of Computer Science and Technology, ISSN : 2229 - 4333, IJCST Vol. 1, Issue 2, December 2010.

[6] Kumar Parasuraman, Member, IEEE and P.Vasantha Kumar, "An Efficient Method for Indian Vehicle License Plate Extraction and Character Segmentation", IEEE International Conference on Computational Intelligence and Computing Research,2010.

[7] Muhammad H Dashtban, Zahra Dashtban, Hassan Bevrani, "A Novel Approach for Vehicle License Plate Localization and Recognition", International Journal of Computer Applications (0975 - 8887), Volume 26- No.11, July 2011.

[8] Stuti Asthana, Niresh Sharma, Rajdeep Singh, "Vehicle number plate recognition using multiple layer back propagation neural networks", International Journal of Computer Technology and Electronics Engineering (IJCTEE), Volume 1, Issue 1, July 10, 2011.

[9] Chetan Sharma1 and Amandeep Kaur2, “ INDIAN VEHICLE LICENSE PLATE EXTRACTION AND SEGMENTATION", International Journal of Computer Science and Communication, Vol. 2, No. 2, pp. 593-599, JulyDecember 2011.

[10] R. T. Lee, K. C. Hung, and H. S. Wang,"Real Time Vehicle License Plate Recognition Based on 2D Haar Discrete Wavelet Transform", International Journal of Scientific \& Engineering Research, Volume 3, Issue 4, ISSN 2229-5518, April-2012.

[11] Lekhana G.C, M.Tech; R.Srikantaswamy, Professor,"Real time license plate recognition system", International Journal of Advanced Technology \& Engineering Research (IJATER) National Conference on Emerging Trends in Technology (NCET-Tech) ISSN, Volume 2, Issue 4, ISSN No: 2250-3536, July 2012.

[12] Wengang Zhou, Houqiang Li, Yijuan Lu, Member, IEEE, and Qi Tian, Senior Member, IEEE, "Principal Visual Word
Discovery for Automatic License Plate Detection", IEEE transaction on image processing ,vol21, NO. 9, September 2012.

[13]P.Sandhya Rani1, Vara Prasad,"License plate character segmentation based on pixel distribution density", [IJESAT] International journal of engineering science and advanced technology, Volume-2, Issue-5, 1539 - 1542, Sep-Oct 2012.

[14] R.Radha1 and C.P.Sumathi2, "A Novel approach to extract text from license plate of vehicle", Signal \& Image Processing: An International Journal (SIPIJ) Vol.3, No.4, and August 2012

\section{BIOGRAPHIES:}

Rinku K. Solanki, M.tech student (digital communication), NRI-IST, Bhopal, Madhya Pradesh, India

Rinkusolanki86@gmail.com

RAJESH KUMAR RAI, Prof.(Head of the department of E.C.), NIRT, Bhopal, Madhya Pradesh, India raj.rai1008@gmail.com

Teena raikwar, SAssistant professor, electronica \& communication dept., (NRI-IST, Bhopal), Madhya Pradesh, India

t_raikwar.2006@yahoo.co.in 